\title{
Therapieoptionen bei kindlichem Asthma
}

Ventolair ${ }^{\odot}$ für Kinder ab 5 Jahren

Mit Ventolair ${ }^{\circledR}$ von 3 M Medica steht erstmals ein inhalatives Kortikoid zur Verfügung, bei dem durch eine innovative Galenik der Wirkstoff Beclometason auch in die kleinen Atemwege gelangt.

Das seit rund einem Jahr erhältliche Präparat verfügt über eine sehr gute therapeutische Wirksamkeit, wobei eine Dosisreduktion um bis zu 50\% bei guter Verträglichkeit gegenüber herkömmlichen beclometasonhaltigen Arzneimitteln möglich wird. Ventolair ${ }^{\circledR}$ erhielt deshalb jetzt auch die Zulassung für die Behandlung von Kindern $a b 5$ Jahren.

Zusätzlich profitieren die Kinder bei der Therapie mit Ventolair ${ }^{\circledR}$ im Autohaler ${ }^{\circledR}$ von der einfachen Handhabung dieser Inhalierhilfe, bei der ein Sprühstoß automatisch zum richtigen Zeitpunkt ausgelöst wird und Anwendungsfehler dadurch vermieden werden. Die Verwendung eines Spacers erübrigt sich.

\section{Asthma: Eltern nur schlecht informiert}

Hier kann eine neue Galenik für den bewährten Wirkstoff Beclometason hilfreich sein: In dem Präparat Ventolair $^{\circledR}$ liegt Beclometason durch Verwendung des FCKW-Ersatztreibgases HFA (Hydrofluoralkan) in gelöster Form vor und gelangt mit den feinen Sprühpartikeln auch in die kleinen Atemwege.

In einer internationalen Studie an über 400 Kindern mit einem Asthma bronchiale konnte gezeigt werden, dass die um den Faktor 3 bis 4 erhöhte Beclometason-Deposition in der Lunge bei reduzierter Dosierung des Pharmakons ein verbesserter klinischer Effekt erreicht wird. Nebenwirkungen werden dadurch reduziert. Die empfohlene Dosierung für Kinder von 5 bis 11 Jahren liegt bei einer Tagesdosis von $100 \mu \mathrm{g}$, in schweren Fällen können auch $200 \mu \mathrm{g} / \mathrm{d}$ gegeben werden.

\section{Kindgerechte Inhalierhilfen}

Gerade bei Kindern muss auch auf eine einfach zu handhabende Inhalationshilfe geachtet werden. Außer Spacern oder atemzugausgelösten Pulverinhalatoren werden dazu jetzt auch atemzuggesteu-

\section{Eine Umfrage ergab, dass viele Eltern asthmakranker Kinder immer noch nicht genügend über den Gebrauch der verordneten Medika- mente informiert sind.}

$\mathrm{E}^{\mathrm{in}}$ ne frühzeitige therapeutische Intervention könnte die Progredienz des Asthma vor allem bei Kindern erheblich verlangsamen. Die Crux: Wir verfügen zwar über geeignete Medikamente, aber die Eltern wissen immer noch zu wenig über den richtigen Einsatz.

Dies ergab jetzt erneut eine Umfrage unter 631 Eltern von Kindern mit Asthma, die vom Londoner Marktforschungsinstitut MORI mit Unterstützung der Firma MSD in 5 europäischen Ländern durchgeführt wurde.

Bei der Frage, was unter einer "Controller"-Therapie zu verstehen sei, wussten z.B. in Deutschland nur rund die Hälfte der Befragten, dass diese Behandlung täglich durchgeführt werden muss, um die Krankheit aufzuhalten. Nur $40 \%$ der Befragten konnten versichern, dass ihr Kind regelmäßig Asthmamedikamente einnimmt und im- merhin noch 25\% machten sich Sorgen wegen der „schädlichen Langzeiteffekte" von Asthmamedikamenten.

Asthma-Aufklärungsprogramme sind also wichtig, um das Therapieproblem Asthma bei Kindern in den Griff zu bekommen. J. Bousquet (Montpellier) appellierte daher: „Wenn die Asthma-Gesundheitsprogramme efolgreich umgesetzt werden und den Eltern ein besseres Wissen vermittelt wird, nehmen Sterblichkeit und Häufigkeit von Klinikeinweisungen wegen Asthma ab selbst wenn die Häufigkeit asthmatischer Erkrankungen insgesamt zunimmt."

\section{Bousquet J}

Pressekonferenz der Firma MSD im Rahmen des Annual Meeting of the European Academy of Allergology and Clinical Immunology, Brüssel/Belgien, 1999. 